\title{
Implementation and Performance Evaluation of a Broadband Digital Harmonic Vector Voltmeter
}

\author{
Domenico Mirri, Gaetano Pasini, Lorenzo Peretto, Fabio Filicori, Gaetano Iuculano, and Andrea Dolfi
}

\begin{abstract}
A broadband digital harmonic vector voltmeter proposed previously and studied theoretically by the authors was implemented using a special-purpose, random sampling strategy, to avoid the bandwidth limitations due to the finite conversion time of the sample-and-hold and analog-to-digital-conversion (S/H-ADC) devices. The experimental results have shown that the bandwidth of the instrument is not limited by the finite conversion time of S/H-ADC devices, since good accuracy can be achieved even when the average sampling frequency is much lower than the signal bandwidth. The amplitude and phase uncertainty, with sinusoidal test signals up to $1 \mathrm{MHz}$ and an average sampling rate of $10 \mathrm{kHz}$, was found to be lower than $3 \%$ and 0.03 rad, respectively. For more careful testing of the broadband performance of our instrument, we also carried out two-frequency, variable order harmonic measurements, which showed good accuracy (amplitude error less than $1.5 \%$ and phase error less than 0.03 rad) with harmonics up to $300 \mathrm{kHz}$. Reasonable accuracy (i.e., sufficient to correctly reconstruct the actual signal waveform) was also found with a highly distorted square-wave signal.
\end{abstract}

Index Terms-Harmonic analysis, nonlinear systems, random variables, sampling methods, voltmeters.

\section{INTRODUCTION}

$\mathbf{T}$ HIS paper describes the implementation of a broadband digital harmonic vector voltmeter proposed previously and studied theoretically by the authors [1]. The measurement procedure uses a digital signal processing algorithm aimed at extracting one of the two periodic exponential components of a sinusoidal reference signal. By time-domain averaging of the product of the signal $s(t)$ and the $n$th power of the reference exponential term, the relative amplitude and phase of any $n$th harmonic of the given signal, normalized with respect to the given sinusoidal reference, can be obtained. For the digital implementation of this instrument it is important to choose a suitable sampling strategy. Thus, to avoid any bandwidth limitation due to a finite sampling frequency, an asynchronous random sampling strategy previously proposed by the authors was used [2].

The measurement procedure is briefly recalled in Section II; the hardware structure of the instrument is described in Section III, while the experimental results, in terms of

Manuscript received May 1, 1997; revised June 1, 1998.

D. Mirri, G. Pasini, L. Peretto, and A. Dolfi are with the Dipartimento di Ingegneria Elettrica, Facoltà di Ingegneria, 40136 Bologna, Italy (e-mail: pasini@eleib1.ing.unibo.it).

F. Filicori is with the Dipartimento di Elettronica, Facoltà di Ingegneria, 40136 Bologna, Italy.

G. Iuculano is with the Dipartimento di Elettronica, Facoltà di Ingegneria, 50139 Firenze, Italy.

Publisher Item Identifier S 0018-9456(98)05491-6.

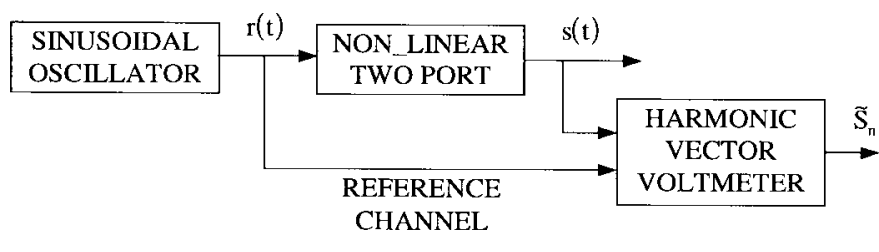

Fig. 1. Block diagram of the measurement system.

magnitude and phase accuracy as functions of frequency, are given in Section IV.

\section{Measurement Procedure}

The harmonic vector voltmeter implemented provides amplitude and phase measurements of the spectral components of a periodic signal $s(t)$ with respect to a sinusoidal reference; in particular, the instrument evaluates the spectral components whose frequencies are integer multiples of that of the reference signal $r(t)$. This instrument can be used, for example, to evaluate the response of a nonlinear system to a sinusoidal signal applied to its input, if this is assumed as the reference signal (Fig. 1).

The periodic signal $s(t)$ can be represented by a Fourier series

$$
s(t)=\sum_{n=-\infty}^{+\infty} S_{n} e^{j n \omega_{1} t}
$$

while the associated sinusoidal reference signal is:

$$
r(t)=A_{r} \frac{e^{j \omega_{1} t}+e^{-j \omega_{1} t}}{2} .
$$

Once the reference exponential term $e^{j \omega_{1} t}$ is known, each Fourier coefficient $S_{n}$ can be derived as the average of the product of $s(t)$ with the $n$th power of $e^{j \omega_{1} t}$, according to the well-known formula

$$
S_{n}=\frac{1}{T_{1}} \int_{-T_{1} / 2}^{T_{1} / 2} s(t) e^{-j n \omega_{1} t} d t
$$

In order to deduce the reference exponential term $e^{j \omega_{1} t}$ from $r(t)$, a signal $r(t-\delta)$ with a prefixed delay $\delta$ can be introduced [1]. In fact we may write

$$
e^{-j \omega_{1} t}=\frac{r(t)}{A_{r}}-j \frac{r(t-\delta)-r(t) \cos \left(\omega_{1} \delta\right)}{A_{r} \sin \left(\omega_{1} \delta\right)} .
$$

In the particular case of $\omega_{1} \delta=(2 m-1)(\pi / 2)$, with $m$ integer, (4) coincides with Euler's formula. Since the frequency $f_{1}$ 
of the reference signal is only approximately known, the quantities $\cos \left(\omega_{1} \delta\right)$ and $\sin \left(\omega_{1} \delta\right)$ must be estimated. It can easily be shown [3] that $\cos \left(\omega_{1} \delta\right)$ can be deduced from the autocorrelation of the signal $r(t)$. For the digital implementation of its autocorrelation, the signal $r(t)$ is sampled at a sequence of successive pairs of instants $\left(t_{w-i}, t_{w-i}-\delta\right)$ and the quantity $\cos \left(\omega_{1} \delta\right)$ is estimated with a moving average filter

$$
\widetilde{\cos }\left(\omega_{1} \delta\right)=\frac{2}{N_{1} A_{r}^{2}} \sum_{i=0}^{N_{1}-1} r\left(t_{w-i}\right) r\left(t_{w-i}-\delta\right)
$$

where $w$ is an integer which marks a specific value of $\widetilde{\cos }\left(\omega_{1} \delta\right)$ deriving from a given set of sampling instants. Thus, the periodic exponential signal can be estimated by the following expression:

$$
\tilde{e}^{-j \omega_{1} t}=\frac{r(t)}{A_{r}}-j \frac{r(t-\delta)-r(t) \widetilde{\cos }\left(\omega_{1} \delta\right)}{A_{r} \sin \left(\widetilde{\omega_{1} \delta}\right)} .
$$

To make the contribution of $\sin \left(\widehat{\omega_{1} \delta}\right)$ to the error negligible, and minimizing the corresponding error [1], a target value

$$
\omega_{1} \delta=(2 m-1) \frac{\pi}{2}, \text { with } m \text { integer }
$$

should be approached. Since the delay $\delta$ is obtained through a programmable counter, $\widetilde{\cos }\left(\omega_{1} \delta\right)$ can assume only a set of discrete values and the condition $\widetilde{\cos }\left(\omega_{1} \delta\right)=0$ in general can only be approached. A suitable $\delta$ is obtained by adjusting its value through a recursive procedure until $\widetilde{\cos }\left(\omega_{1} \delta\right)(5)$ verifies the condition (7) with an approximation such as to guarantee $\sin \left(\widehat{\omega_{1} \delta}\right) \cong 1$.

An estimate of the Fourier series coefficients $S_{n}$ of $s(t)$ can be digitally determined with a moving average of the last $N_{2}$ successive values of the sampled signal $s(t)$ multiplied by the $n$th power of $\tilde{e}^{-j \omega_{1} t}[1]$

$$
\tilde{S}_{n}=\frac{1}{N_{2}} \sum_{i=0}^{N_{2}-1} s\left(t_{k-i}\right) \tilde{e}^{-j n \omega_{1} t_{k-i}}
$$

where $k$ is an integer which marks a generic time-dependent value of $\widetilde{S}_{n}$.

In order to overcome the bandwidth limitation due to the finite sampling speed, a convenient random sampling strategy was used [2], [4]-[6]. In this way, the only bandwidth limitation of the instrument derives from the bandwidth of the $\mathrm{S} / \mathrm{H}$ circuit. This strategy divides the time axis into successive equal intervals $T_{c}$; within each of these intervals a random instant is inserted with a continuous uniform distribution in the interval. More precisely, a generic sampling instant can be defined as follows:

$$
\begin{aligned}
& t_{k-i}=\tau_{0}+\left(k-i+X_{k-i}\right) T_{c} \quad \text { with } \\
& i=0 \cdots N_{2} \cdots N_{2}+N_{1}-1
\end{aligned}
$$

where the unknown constant $\tau_{0}$ represents the initial shift between the origin of the sampling sequence and the reference signal $r(t)$; $T_{c}$ is the mean sampling-time interval, while $X_{k-i}$ is the $i$ th component of a set of random independent variables having a common continuous uniform distribution in the interval $\pm a$, with $a \approx 1 / 2$. The sequences of the random

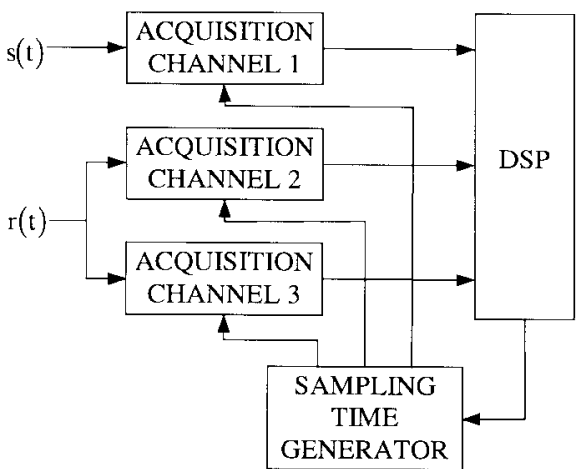

Fig. 2. Simplified block diagram of the digital harmonic vector voltmeter.

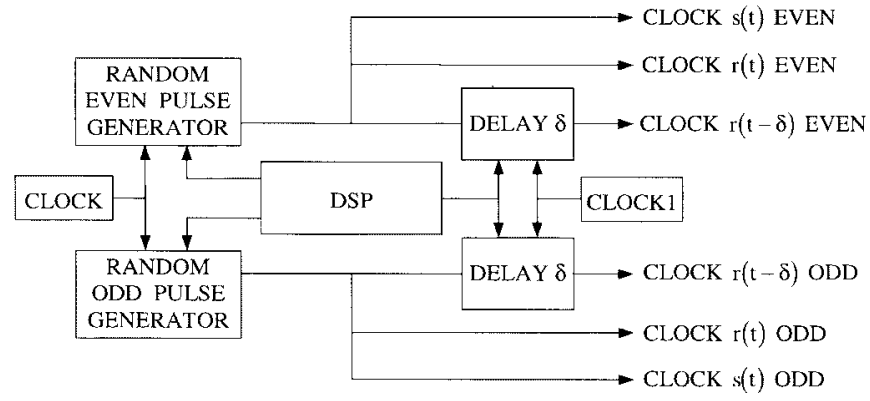

Fig. 3. Block diagram of the sampling time generator.

instants used to estimate $\cos \left(\omega_{1} \delta\right)$ and $S_{n}$ are different in order to guarantee that they are estimated independently.

\section{HARDWARE STRUCTURE}

Fig. 2 shows a simplified block diagram of the harmonic vector voltmeter. The selected random sampling strategy implies that the time interval between two adjacent sampling instants can be practically null, while the distance of the following one is not smaller than $T_{c}$; thus, to satisfy these conditions each of the three identical acquisition channels includes two identical acquisition systems which operate, respectively, on alternate sampling instants [e.g., odd and even $i$ in (9)]. With reference to Fig. 2, the periodic signal $s(t)$ is applied to the first input module (called channel 1), while the reference signal $r(t)$ and the delayed ${ }^{1}$ one $r(t-\delta)$ are applied to the second and the third modules (channel 2 and channel 3), respectively. The timing signals, required by each of the three acquisition channels according to the adopted random asynchronous sampling strategy, are generated by a suitable sampling time generator circuit. Fig. 3 shows the block diagram of the sampling time generator. This generator is synchronized with a "master clock" which divides the time axis into equally spaced intervals; within each interval a random sampling instant must be provided. For this purpose, a random number of eight bits is generated by the DSP. This number is first converted into a corresponding voltage amplitude by a DAC and then into a time instant by comparing it with a voltage ramp signal starting at the beginning of each

\footnotetext{
${ }^{1}$ Actually no delay in $r(t)$ is introduced, but an equivalent delay difference in the sampling time is introduced by the sampling time generator (see Fig. 3).
} 
time interval. When the amplitudes of the two signals coincide, a sampling pulse is generated. To achieve a continuous random distribution of the sampling instants, a random white noise of adequate amplitude is added to the output of the DAC.

First, the amplitude $A_{r}$ of the reference signal $r(t)$ is determined measuring its rms value. The required rms value can be estimated by means of the simple formula

$$
\tilde{A}_{r}=\sqrt{\frac{1}{N} \sum_{i=0}^{N-1} r^{2}\left(t_{k-i}\right)}
$$

using the adopted asynchronous random sampling strategy [2], [4]-[6]. Then, implementing (5) by software, a suitable value of $\delta$, giving a sufficiently small $\left|\widetilde{\cos }\left(\omega_{1} \delta\right)\right|$ must be found [in our testing procedures, the condition $\left|\widetilde{\cos }\left(\omega_{1} \delta\right)\right|<0.05$ is imposed as a criterion for the search of a convenient value for $\delta]$.

As the frequency of the clock signal which drives the delay generators is $f_{c 1}=10 \mathrm{MHz}$, the delay $\delta$ can be increased in steps of $100 \mathrm{~ns}$. The final value of $\delta$ can be obtained by adopting a convenient recursive procedure, which assures an adequate resolution and simultaneously optimizes the search and lock time, . Then this value can be used to estimate, through (6), the periodic exponential signal corresponding to the reference signal $r(t)$, in order to evaluate the spectral components of the signal $s(t)$ by software implementing (8).

Each channel can accept an input signal in the range \pm 10 V. Each module contains the S/H HA-5340 device (gainbandwidth product $10 \mathrm{MHz}$, slew rate $40 \mathrm{~V} / \mu \mathrm{s}$ for $10 \mathrm{~V}$ output step) and the ADC HI 674-A (12 bits, conversion time 15 $\mu \mathrm{s})$, both manufactured by Harris. The average sampling-time interval is $T_{c}=100 \mu$ s (i.e., the mean sampling frequency is as low as $f_{c m}=10 \mathrm{kHz}$ ).

Since the proposed harmonic vector voltmeter involves computationally intensive data processing algorithms, a floating point DSP located on a board connected to a PC was used. The communication with the external peripherals was implemented by means of a DSP-Link bus.

\section{MeAsurement Results}

The instrument calibration was performed only in dc conditions, by comparison with the HP3458A multimeter and according the manufacturer's suggested procedure, which requires trimming the offset and gain of the ADC until the total $\mathrm{dc}$ error becomes less than $\pm 3 \mathrm{mV}$. The number of successive sampled values of $r(t)$ and $r(t-\delta)$ used in the moving average filter to estimate $\cos \left(\omega_{1} \delta\right)$ through (5) was $N_{1}=2^{13}$. Also the values of $N_{2}$ in (8) and $N$ in (10) were selected equal to $2^{13}$. To reduce the effect of the noise introduced by the acquisition channels and the variance associated to the sampling strategy [2], [4]-[6], the mean value of twenty successive measurements of $\widetilde{S}_{n}$ was considered.

The frequency response of the harmonic vector voltmeter was evaluated for three different phase values of an input sinusoidal signal $s(t)$ with respect to the reference one $r(t)$, as shown in Table I. Both these signals were generated by the two-channel synthesizer HP3326A, which also allows
TABLE I

Magnitude and Phase Accuracy for a Sinusoidal Test Signal with Three Different Phase Values with Respect to the Reference One

\begin{tabular}{c|c|c|c|c}
\hline $\begin{array}{c}\text { Frequency } \\
(\mathrm{kHz})\end{array}$ & $\begin{array}{c}\text { Phase } \varphi_{1} \\
(\mathrm{rad})\end{array}$ & $\Delta \mathrm{S}_{1}(\%)$ & $\Delta\left(\frac{\mathrm{S}_{1}}{\mathrm{~A}_{\mathrm{r}}}\right) \%$ & $\Delta \varphi_{1}(\mathrm{rad})$ \\
\hline 4 & 0 & .3 & 0.50 & .0002 \\
& $\pi / 2$ & -2.2 & & -.04 \\
& $(3 / 4) \pi$ & -2.0 & & .04 \\
\hline 8 & 0 & -.5 & 0.58 & .003 \\
& $\pi / 2$ & -1.3 & & -.06 \\
& $(3 / 4) \pi$ & -.9 & & .12 \\
\hline 16 & 0 & 1.3 & 2.5 & .004 \\
& $\pi / 2$ & .02 & & -.007 \\
& $(3 / 4) \pi$ & -.2 & & .03 \\
\hline 32 & 0 & -.6 & 1.2 & .02 \\
& $\pi / 2$ & -.9 & & .005 \\
& $(3 / 4) \pi$ & -1.1 & & .03 \\
\hline 64 & 0 & -.5 & 2.4 & .008 \\
& $\pi / 2$ & -.6 & & .007 \\
& $(3 / 4) \pi$ & -.2 & & .02 \\
\hline 128 & 0 & -.1 & 1.4 & -.01 \\
& $\pi / 2$ & -.7 & & .009 \\
& $(3 / 4) \pi$ & -.3 & & -.002 \\
\hline 256 & 0 & -.8 & 2.4 & .02 \\
& $\pi / 2$ & -1.2 & & -.01 \\
& $(3 / 4) \pi$ & -1.3 & & .007 \\
\hline 512 & 0 & .5 & 2.1 & .04 \\
& $\pi / 2$ & -.6 & & .003 \\
& $(3 / 4) \pi$ & .7 & & -.009 \\
\hline 1024 & 0 & -.8 & 2.9 & -.008 \\
& $\pi / 2$ & -.6 & & -.003 \\
& $(3 / 4) \pi$ & -1.2 & & .02 \\
\hline & & & &
\end{tabular}

adjusting the phase difference between the two channels, once a calibration procedure to set the null phase value has been carried out; to this end the digital oscilloscope TK2440 was used.

The amplitudes of $r(t)$ and $s(t)$ were measured with two HP3458A multimeters. If we recall that the value of the average sampling frequency $f_{c m}$ was only $10 \mathrm{kHz}$, the results confirm that the adopted random asynchronous sampling strategy is such as to overcome any bandwidth limitation deriving from the finite sampling rate [2], [3]-[5]. Thus, the instrument bandwidth practically depends only on the bandwidth of the $\mathrm{S} / \mathrm{H}$ adopted in our implementation (i.e., $10 \mathrm{MHz}$ gainbandwidth product and a maximum slew-rate of $40 \mathrm{~V} / \mu \mathrm{s}$ in large signal operation). As regards the magnitude accuracy, we obtained an absolute accuracy $\Delta S_{1} \leq 2.2$ and a ratio accuracy $\Delta\left(S_{1} / A_{r}\right) \leq 3$; the phase discrepancy was less than $0.02 \mathrm{rad}$. In order to test the harmonic vector voltmeter accuracy under periodic, nonsinusoidal operation, different two-frequency signals, including a fundamental and different order harmonics, were used. These two frequency signals were generated using the two-channel synthesizer HP 3326A, while the reference signal was provided by a third function generator synchronized with the HP 3326A. The amplitude of both the fundamental and the harmonic was $2 \mathrm{~V}$. The results given in Table II show good overall accuracy (better than $1.5 \%$ for the amplitude and $0.03 \mathrm{rad}$ for the phase). This confirms the broadband capabilities of the signal sampling and numerical processing procedures adopted. In fact, both the fundamental and harmonic frequencies are well above not only the average sampling frequency adopted $(10 \mathrm{kHz})$ but also the maximum 


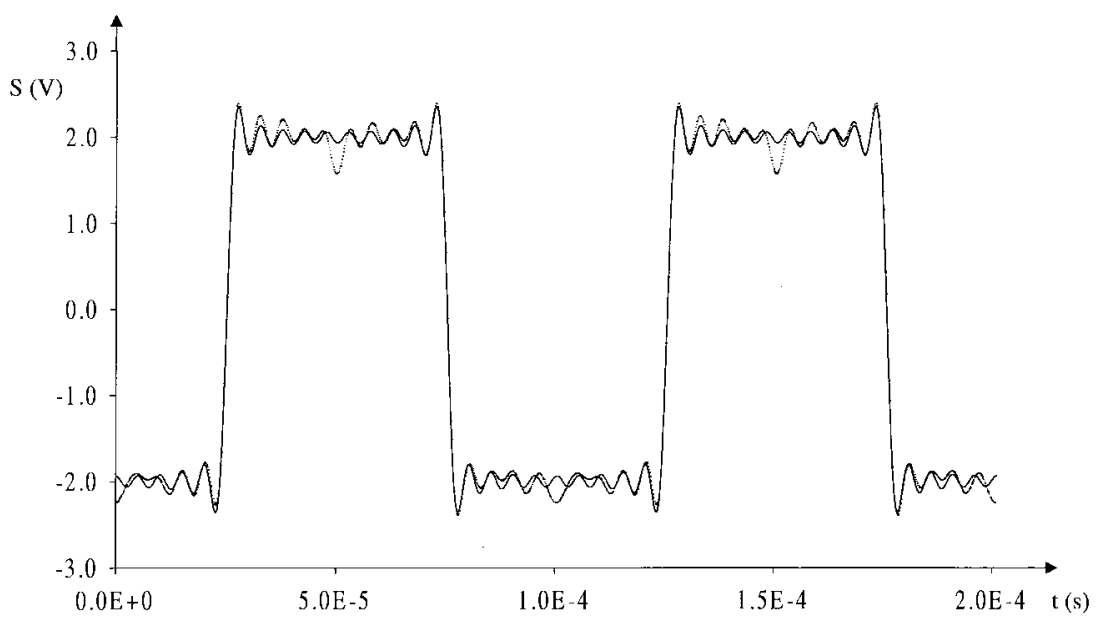

Fig. 4. Comparison between the theoretical shape of a square wave with only the first twenty harmonic components (continuous line) and the experimental one obtained with the measured values (dotted line).

TABLE II

Amplitude and Phase ERror of the SPeCtral Components of Different Signals $s(t)$ with the SAme Fundamental Frequency $\left(S_{n}=2 \mathrm{~V}\right)$

\begin{tabular}{c|c|c}
\hline $\begin{array}{c}\text { Frequency of the } \\
\text { spectral components }\end{array}$ & $\Delta \mathrm{S}_{\mathrm{n}}(\mathrm{V})$ & $\Delta \varphi(\mathrm{rad})$ \\
\hline $\mathrm{f}_{1}=62.5 \mathrm{kHz}$ & .02 & -.02 \\
\hline $\mathrm{f}_{1}=62.5 \mathrm{kHz}$ & .007 & -.001 \\
$2 \mathrm{f}_{1}$ & -.03 & .001 \\
\hline $\mathrm{f}_{1}=62.5 \mathrm{kHz}$ & -.01 & .002 \\
$3 \mathrm{f}_{1}$ & -.03 & -.01 \\
\hline $\mathrm{f}_{1}=62.5 \mathrm{kHz}$ & .006 & -.005 \\
$4 \mathrm{f}_{1}$ & .02 & -.03 \\
\hline $\mathrm{f}_{1}=62.5 \mathrm{kHz}$ & -.01 & .02 \\
$5 \mathrm{f}_{1}$ & -.01 & .004 \\
\hline
\end{tabular}

sampling frequency which, in a conventional sampling approach, would be allowed by a $15 \mu$ s conversion time of the HI 674-A ADC device. The performances discussed above were achieved by designing our prototype instrument according to conventional analog and digital design techniques, by simply following the state-of-the-art and, in particular, the component manufacturer's recommendations. In this respect, it should be noted that the nonideal behavior of most of the many building blocks in Fig. 1 (e.g., all the components of the sampling time generator, except for the delay blocks) is not critical for the instrument accuracy, since the only requirement on these elements is producing a pseudorandom set of sampling instants with a reasonably uniform distribution. Actually, a good approximation of the required uniform, pseudorandom sampling time distribution was achieved; this was verified by measuring the sequence of sampling instants by means of an HP 3470B universal time interval counter. Good uniformity of the sampling time distribution was then confirmed by the Kolmogorov-Smirnov test. In fact, the maximum absolute difference between the empirical distribution function and the assumed uniform one was less than $5 \times 10^{-3}$. For the test at the $1 \%$ significance level the critical value for $n=2^{15}$ data is $1.6276 / \sqrt{n}=9.0 \times 10^{-3}$, which is greater than the observed value $5 \times 10^{-3}$. Consequently, our actual significance level
TABLE III

Theoretical and Measured Amplitudes and Phases of the First Twenty Spectral Components of a Square Wave Signal

\begin{tabular}{|c|c|c|c|c|}
\hline $\begin{array}{l}\text { Frequency of } \\
\text { the spectral components }\end{array}$ & $\left|S_{n}\right|(V)$ & $\left|\tilde{S}_{n}\right|(V)$ & $\varphi_{\mathrm{n}}(\mathrm{rad})$ & $\bar{\varphi}_{\mathrm{n}}(\mathrm{rad})$ \\
\hline$f_{1}=62.5 \mathrm{kHz}$ & 2.546 & 2.554 & 3.142 & 3.134 \\
\hline $2 \mathrm{f}_{1}$ & 0 & 0.045 & - & - \\
\hline $3 \mathrm{f}_{1}$ & 0.849 & 0.868 & 0 & -0.024 \\
\hline $4 f_{1}$ & 0 & 0.046 & - & - \\
\hline $5 f_{1}$ & 0.509 & 0.500 & 3.142 & 3.124 \\
\hline $6 \mathrm{f}_{1}$ & 0 & 0.039 & - & - \\
\hline $7 f_{1}$ & 0.364 & 0.369 & 0 & -0.082 \\
\hline $8 f_{1}$ & 0 & 0.035 & - & - \\
\hline $9 f_{1}$ & 0.283 & 0.277 & 3.142 & 3.028 \\
\hline $10 f_{1}$ & 0 & 0.036 & - & - \\
\hline $11 f_{i}$ & 0.231 & 0.229 & 0 & -0.081 \\
\hline $12 \mathrm{f}_{1}$ & 0 & 0.036 & - & - \\
\hline $13 f_{1}$ & 0.195 & 0.198 & 3.142 & 2.926 \\
\hline $14 f_{1}$ & 0 & 0.038 & - & - \\
\hline $15 f_{1}$ & 0.170 & 0.171 & 0 & -0.131 \\
\hline $16 f_{1}$ & 0 & 0.035 & - & - \\
\hline $17 f_{1}$ & 0.150 & 0.156 & 3.142 & 2.960 \\
\hline $18 f_{1}$ & 0 & 0.031 & - & - \\
\hline $19 f_{1}$ & 0.134 & 0.134 & 0 & -0.218 \\
\hline $20 f_{1}$ & 0 & 0.030 & - & - \\
\hline
\end{tabular}

exceeds $1 \%$ and we may regard the data as consistent with the hypothesis that they come from a uniform distribution.

Delay block implementation, on the other hand, can be more critical for spectral analysis accuracy, since delay errors involve inaccuracies in the extraction of the reference exponential term of (6), as shown in [1]. As delay was implemented through a programmable counter with $100 \mathrm{~ns}$ resolution, this uncertainty (which could possibly be improved) is probably one of the causes of the accuracy limits found in the measurements.

Finally, in order to check the instrument's capability to deal with realistic, broadband periodic signals, the first twenty spectral components of a $62.5 \mathrm{kHz}$ symmetric square wave with respect to a synchronous sinusoidal signal assumed as 
reference were measured. These harmonics were compared with the theoretical values corresponding to an ideal squarewave signal characterized by the same true rms value $\left(S_{\text {rms }}=\right.$ $2 \mathrm{~V}$ ) which was measured with the HP3458A multimeter.

No direct comparison with the amplitude and phase spectrum of the nonideal square-wave provided by the HP 3326A synthesizer was possible, since a vector spectrum analyzer with adequate accuracy and bandwidth was not available. Thus, the amplitude and phase discrepancies in Table III include both the instrument's inaccuracies and test signal nonidealities. An overall evaluation of the instrument accuracy in the analysis of a highly distorted broadband signal, like a square wave, can be based on the global rms relative error

$$
\varepsilon_{R}=\frac{\Delta S_{\mathrm{rms}}}{S_{\mathrm{rms}}}=\sqrt{\frac{\frac{1}{2} \sum_{n=1}^{M}\left|\tilde{S}_{n}-S_{n}\right|^{2}}{S_{\mathrm{rms}}^{2}}} .
$$

In the case of the square wave spectrum given in Table III, the global rms relative error $\varepsilon_{R}$, computed by consider $M=20$ harmonics, was found to be less than $4 \%$, which includes not only instrument inaccuracy but also test-signal nonideality. The reasonably good overall accuracy in the measurement of the amplitude and phase spectra of a square-wave signal is also qualitatively confirmed by the comparison in Fig. 4 between the ideal and measured waveforms plotted by considering $M=20$ harmonics.

The accuracy limits found in the above described tests can be caused by different sources of error, like the finite resolution in the delay block needed for the extraction of the periodic exponential signal from the reference channel, the numerical errors in the DSP algorithm and the noise introduced by the acquisition channels. We have verified that this noise can be reduced by more than one order of magnitude using optical isolators between each acquisition channel and the digital signal processing hardware.

\section{CONCLUSIONS}

The implementation of a digital harmonic vector voltmeter based on a special purpose random sampling strategy has been presented. The prototype instrument, based on a floating point DSP, has been tested on different signals, including a single sinusoid with different phases with respect to the reference one, a sinusoid plus a single harmonic of different order and a square wave. In all cases quite good accuracy both in magnitude and phase was found, even when the signal frequency was much higher than the mean sampling frequency. This feature directly derives from the basic properties of the random sampling strategy adopted and would be practically useful for implementing instruments operating at relatively high frequencies.

\section{ACKNOWLEDGMENT}

The authors gratefully acknowledge the assistance of Dr. Eng. O. Galeotti for designing the instrument, setting the automated measurement system, and carrying out the experimental results.

\section{REFERENCES}

[1] D. Mirri, G. Iuculano, A. Menchetti, F. Filicori, G. Pasini, and M Catelani, "A broad band harmonic vector voltmeter based on a random sampling strategy," in IEEE Instrumentation Measurement Technol. Conf. (IMTC/94), Hamamatsu, Japan, May 1994, pp. 1012-1015.

[2] F. Filicori, G. Iuculano, A. Menchetti, and D. Mirri, "A random asynchronous sampling strategy for measurement instruments based on nonlinear signal conversion," Proc. Inst. Elect. Eng. A, vol. 136, pp. 141-150, May 1989.

[3] W. Mc C. Siebert, Circuits, Signals and Systems. New York: McGrawHill, 1986.

[4] F. Filicori, G. Iuculano, A. Menchetti, D. Mirri, and M. Catelani, "New performance function for the comparison of different sampling strategies in nonlinear conversion instruments," in Proc. IEEE Instrumentation Measurement Technol. Conf., Washington, DC, Apr. 1989, pp. 307-311.

[5] G. Iuculano, D. Mirri, F. Filicori, A. Menchetti, and M. Catelani, "A criterion for the analysis of synchronous and asynchronous sampling instruments based on nonlinear processing," Proc. Inst. Elect. Eng. A, vol. 139, pp. 141-152, July 1992.

[6] D. Mirri, F. Filicori, G. Iuculano, A. Menchetti, and M. Catelani, "Accuracy evaluation of digital wattmeters based on different sampling strategies," in IMEKO TC7 Symp. "Knowledge Based Measurements," Karlsruhe, Germany, Sept. 1990, pp. 117-124.

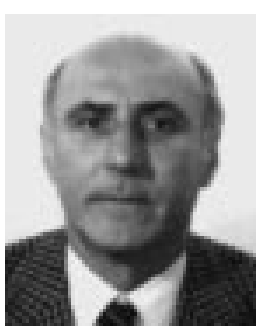

Domenico Mirri was born in Italy in 1936. He graduated in electronic engineering from the University of Bologna, Bologna, Italy.

At present, he is Associate Professor of Electronic Measurement, University of Bologna. His main areas of interest are power and biomedical measurements, digital signal processing in measurement instruments, and characterization of nonlinear systems with memory.

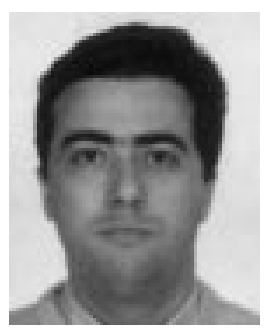

Gaetano Pasini was born in Italy in 1964. He graduated in electronic engineering from the University of Bologna, Bologna, Italy, where he is now a Researcher in Electrical Measurement.

His research activity is mainly oriented to digital signal processing in electronic instruments, power measurements, and characterization of nonlinear systems with memory.

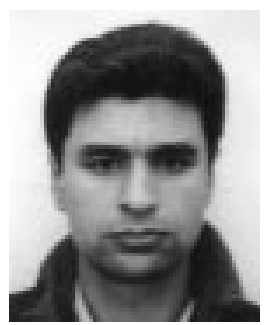

Lorenzo Peretto was born in Italy in 1968 . He graduated in electronic engineering from the University of Bologna., Bologna, Italy. He is currently pursuing the Ph.D. degree in the Department of Electrical Engineering at the University of Bologna.

His field of research is the measurement of power systems under nonsinusoidal conditions.
Fabio Filicori was born in Italy in 1949. He graduated in electronic engineering from the University of Bologna, Bologna, Italy, in 1964.

At present, he is a Full Professor of Applied Electronics at the University of Bologna. His current research interests are in the areas of nonlinear circuit analysis and design, electronic devices modeling, digital measurement instruments, and power electronics. 
Gaetano Iuculano was born in Italy in 1938. He graduated in electronic engineering from the University of Bologna, Bologna, Italy.

At present, he is a Full Professor of Electrical Measurements at the University of Florence, Florence, Italy. His current research interests are calibration applications, reliability analysis and life testing for electronic devices and systems, statistical analysis, and digital measurement instruments.

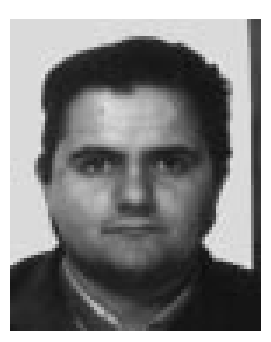

Andrea Dolfi was born in Italy in 1970. He graduated in electronic engineering from the University of Bologna, Bologna, Italy.

His research activity is mainly oriented to digital signal processing for measurement instruments. 